\title{
Using of non-traditional current and voltage sensors for the fault location
}

\author{
Andrey Yablokov ${ }^{1, *}$, Galina Filatova $^{1}$, and Alexander Timofeev ${ }^{1}$ \\ ${ }^{1}$ Ivanovo State Power Engineering University, 153024 Ivanovo, Russia
}

\begin{abstract}
The article describes the possibilities of using the nontraditional current and voltage sensors to solve the problem of fault location in terms of digital transformers developed at the Ivanovo State Power Engineering University (ISPEU). Frequency and metrological characteristics of current and voltage sensors are given.
\end{abstract}

\section{Introduction}

The high-voltage power lines are quite often damaged parts of energy system. Line outage is always accompanied by undersupply of energy, decreasing of reliability, cost and quality of electric supply. That's why one of the important tasks of line maintenance is quick looking for location of the damage and organization of the rehabilitation.

So there are wide-spreading methods and means of remote fault location on the power lines in power industry. Solving of these problems is the one of the most important tasks related with the conception of creating active-adaptive electrical system (Smart Grid). This conception has new such properties as self-diagnosis, self-healing, the ability to identify "weak" parts of the system and change its operating mode, and it helps to prevent the emergence of technological accidents. The development of market relations in the power industry, the requirements to ensure reliable power supply and rapid recovery of power industry connections lead to the need for accurate determination of the fault location and special algorithms.

The development of non-traditional current and voltage sensors (including digital current and voltage transformers) that allow to measure voltage and current without distortion due to lack of enrichment, but with higher accuracy due to exclusion of analogue secondary conduits and isolating transformers, and also having wider frequency ranges [1], involve a significant increase of the accuracy of remote fault location algorithms [2].

Nowadays, with the development of the satellite synchronization technology the interest in pulsed methods of remote fault location based on measurement of the electromagnetic waves' movement time through the power lines increases. The most widespread passive or wave methods are ones that use natural electromagnetic waves which appear in fault location [2]. As the experience in the operation of wave remote fault location shows, the accuracy of fault location without equipment cost's increasing and its operation is about \pm 300 meters (about one span of the line). But, in practice, the accuracy of the fault location

\footnotetext{
* Corresponding author: andrewyablokov@yandex.ru
} 
is significantly influenced by frequency characteristics of primary converters, accuracy in sensor signals, sampling accuracy, synchronization accuracy, accuracy in determination the wave parameters of the power lines and other factors $[4,5]$.

Also widespread methods of remote fault location are based on measurement of the emergency mode parameters (EMP). The advantages of these methods are simplicity of usage, absence of generating equipment, possibility of usage on every kind of lines. Also remote fault location, which based on measuring EMP has large (about 10-20\%) accuracy caused by accuracy of current and voltage sensors $[5,6]$.

So, the development of new remote fault location algorithms based on signals from digital current and voltage transformers and research of accuracy in digital transformers are practical interesting.

\section{Non-traditional current and voltage sensors}

There were produced digital current and voltage transformers, combined transformers for voltage 6 (10), 35, 110 and $220 \mathrm{kV}$ (fig. 1) at the Department of electric power systems automatic control, ISPEU. Nowadays the developed digital transformers are entered in the register measure instruments.

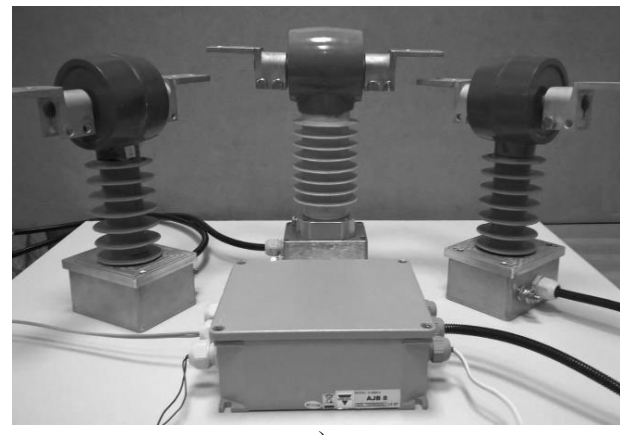

a)

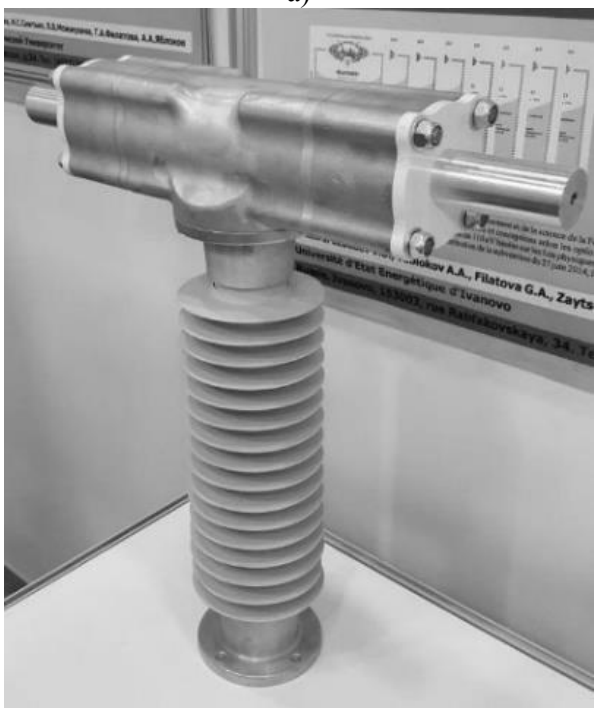

b)

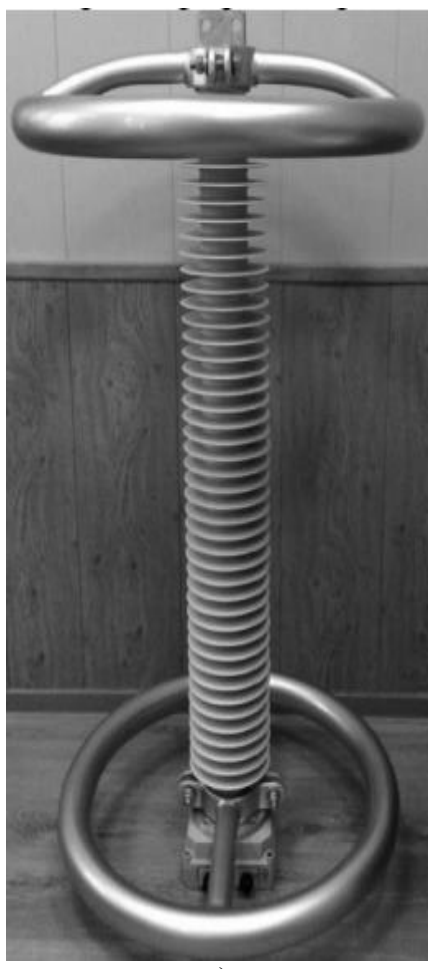

c)

Fig. 1. Experimental samples of digital current and voltage transformers: a) 6 (10) kV; b) $35 \mathrm{kV}$; c) $110 \mathrm{kV}$. 
Primary converters of digital transformers contain several non-traditional current sensors based on different physical principles, and non-inductive voltage dividers. The tests on the laboratory equipment of the ISPEU and the accredited testing center for measuring instruments ("ICRM") showed that it's promising to use digital transformers for the fault location problems because of their better frequency characteristics and smaller accuracy (fig. 2-5).

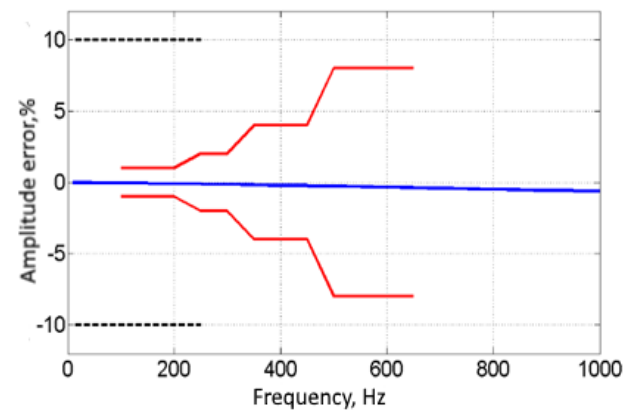

a)

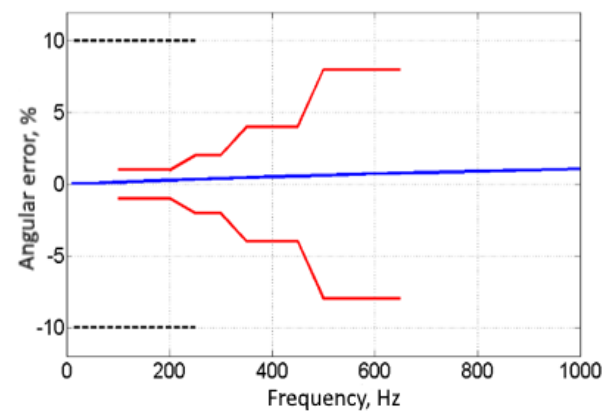

b)

Fig.2. Amplitude-frequency (a) and phase-frequency (b) characteristics of a small-sized current transformer with an accuracy margin for the calculation of electricity and protection for traditional usage in accordance with the Standard IEC 60044-8-2010.

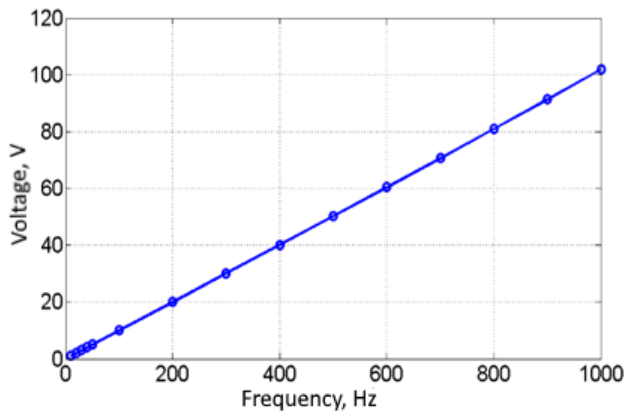

a)

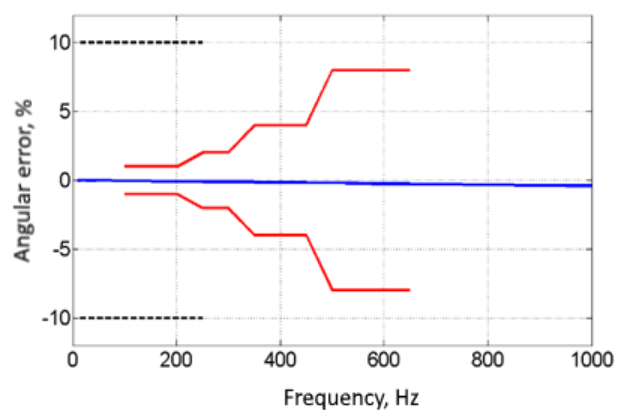

b)

Fig. 3. Dependence of the voltage on the Rogowski belt on frequency (a) and the phase-frequency characteristic (b) with a designation of accuracy limits for the calculation of electricity and protection for traditional usage in accordance with the Standard IEC 60044-8-2010.

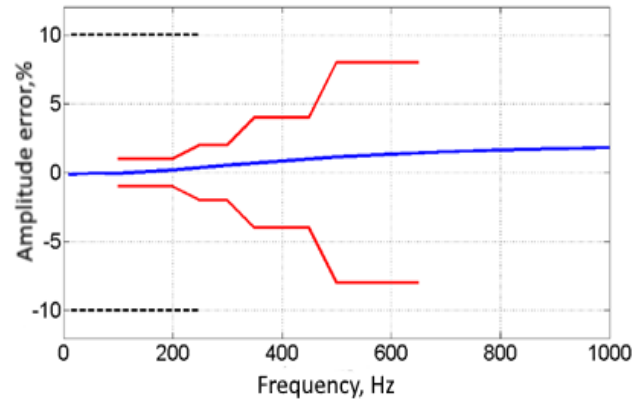

a)

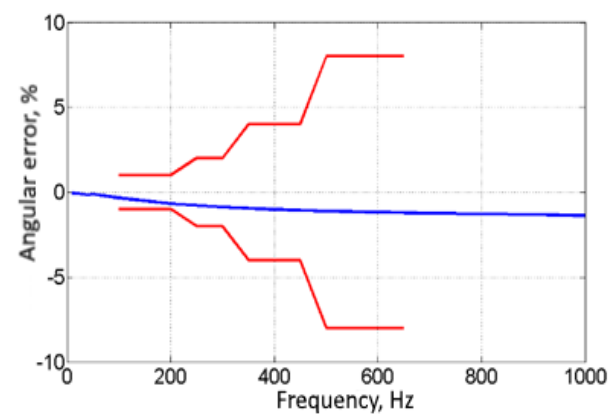

b)

Fig. 4. Amplitude-frequency (a) and phase-frequency (b) characteristics of the direct current sensor with an indication of the accuracy limits for the calculation of electricity and protection for traditional usage in accordance with the Standard IEC 60044-8-2010. 


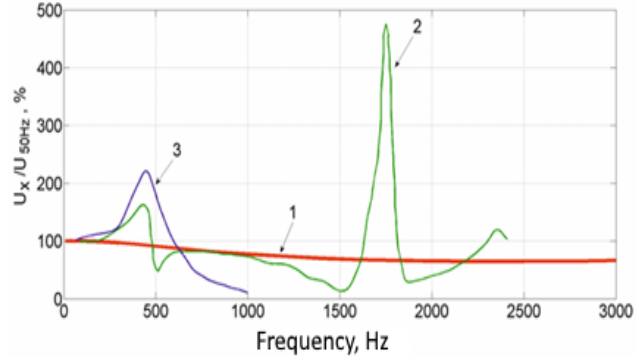

a)

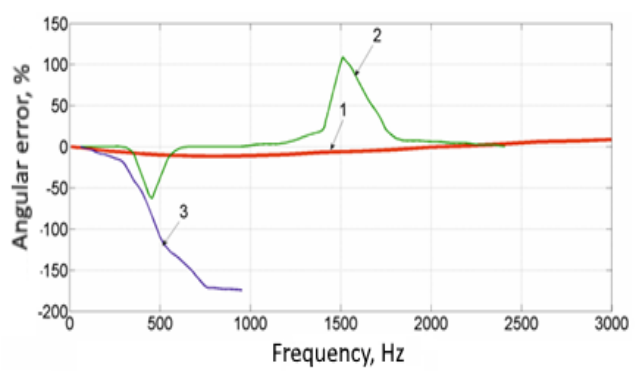

b)

Fig.5. Amplitude-frequency and phase-frequency characteristics: a) primary voltage converter of digital voltage transformer $(110 \mathrm{kV}) 2)$ inductive voltage transformer $(420 \mathrm{kV})$ with oil-and-paper insulation 3) capacitive voltage transformer.

Primary current converters, according to the test results, have a high accuracy in the wide range of currents from 1 to $120 \%$ In and with a reserve are placed within the limits of the accuracy class $0.2 \mathrm{~s}$ with a three-fold overcurrent capability. It has the same results in a wide frequency range. The use of produced digital transformers with improved metrological characteristics in a wide frequency range will increase the accuracy of remote fault location based both on wave methods and on the measurement of EMP.

At the moment one-sided metering algorithms using as input signals currents and voltages obtained with the digital combined transformers are being developed. The algorithm is based on remote metering using the voltage measured from the Rogowski belt, proportional to the derivative of the primary current. Measurements occur at the moments of the transient current crosses zero values measured by a high-precision shunt. Measurement of voltage on the damaged phase is made by a resistive divider. The use of voltage from the Rogowski belt leads to an increase in the accuracy of the measurement compared with the use of the mathematically calculated derivative (fig. 6).
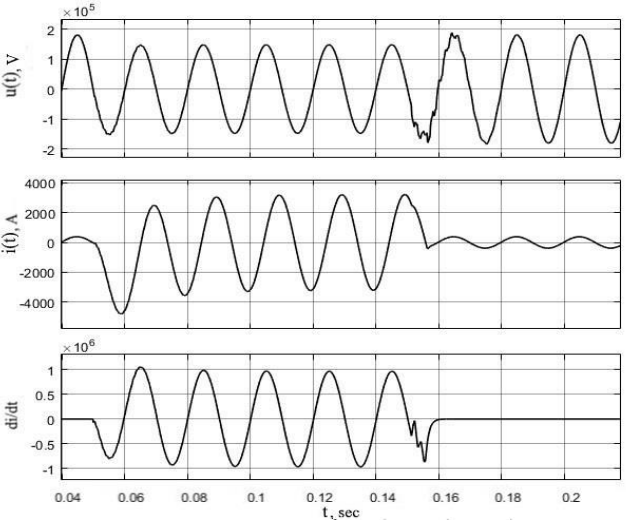

a)

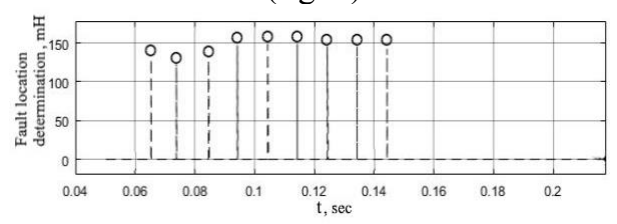

b)

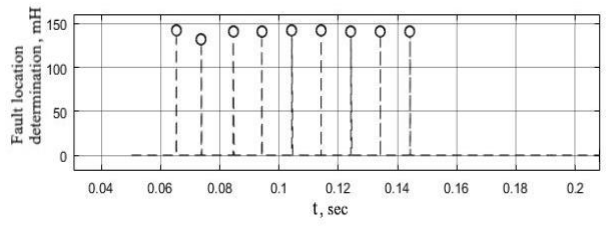

c)

Fig.6. Electrical quantities during short circuit (a) and measured fault location - using mathematically calculated derivative (b) and voltage measured from the Rogowski belt (c), where $150 \mathrm{mH}$ is true short circuit inductivity.

The estimation of the distorting factors (transient resistance in the fault location, load current, capacitive conductivity of the line, accuracy of specifying the specific parameters of the transmission line) is carried out on the models of electric networks in Matlab.

The research was made thanks to the grant from Russian Science Foundation (project No. 17-7910455) at the Ivanovo State Power Engineering University. 


\section{References}

1. V.D. Lebedev, A.A. Yablokov, IOP Conf. Series: Mater. Sci. Eng. 177, 1 (2017), DOI: 10.1088/1757-899X/177/1/012099

2. G. Filatova, V. Lebedev, V. Shuin, ICIEAM, 1 (2016), DOI: 10.1109/ICIEAM.2016.7911011

3. Standard 56947007-29.240.55.224-2016. Studies of fault location determination on overhead power lines voltage $110 \mathrm{kV}$ and more. The standard of company. Introduction date: 17.08.2016 [in Russian]

4. S. Marx, B. K. Johnson, A. Guzman, V. Skendzic, M. V. Mynam, Int. Conf. Power Syst. Transients, 15 (2015)

5. Yu.V. Bychkov, V.N. Koslov, K.I. Ermakov, J. Relay Prot. Electric Power Syst. Automatics, 1, 42 (2016)

6. Otchet po naturnym ispytaniyam ustrojstv OMP VLEHP (Report on tests fault location devices). Report of FGC UES, 04.12.2015. Stream: http://aurae.ru/files/OTЧЕT_OMП.pdf [in Russian] 